\title{
Toward a consensus in the laboratory diagnostics of Fabry disease - recommendations of a European expert group
}

\author{
Andreas Gal • Derralynn A. Hughes • Bryan Winchester
}

Received: 6 September 2010 /Revised: 28 November 2010 / Accepted: 2 December 2010 /Published online: 13 January 2011

(C) The Author(s) 2011. This article is published with open access at Springerlink.com

Guidelines on diagnostics and therapy of Fabry disease have already been compiled in a number of European countries and are being prepared in others. A synthesis of these national guidelines seems to be a sensible option for preparing a prospective European consensus document in the not too distant future. While clinical diagnostics is extensively discussed in the various guidelines, at present there is no consensus on laboratory diagnostic tests for Fabry disease either at national level or at the level of the European Union. There is a widespread variation concerning the diagnostic value of the various methods, such as enzyme activity testing, gene analysis, biopsies, Gb3 measurement etc. and on what should be used and how

Communicated by Douglas A. Brooks

Competing interest: None declared.

This recommendation was compiled by the authors on behalf of the 21 European Experts listed in the article.

\section{A. Gal $(\square)$}

Institut für Humangenetik, Universitätsklinikum

Hamburg-Eppendorf,

Martinistr. 52,

20246 Hamburg, Germany

e-mail: gal@uke.de

D. A. Hughes

Lysosomal Storage Disorders Unit,

Department of Academic Haematology, Royal Free Hospital

\& University College Medical School,

Rowland Hill Street,

London NW3 2PF, UK

e-mail: d.hughes@medsch.ucl.ac.uk

B. Winchester

Biochemistry Research Group, UCL Institute of Child Health at Great Ormond Street Hospital, University College London,

30 Guilford Street,

London WC1N 1EH, UK

e-mail: b.winchester@ich.ucl.ac.uk they compare, if indeed they do. This results in lack of agreement regarding the clinical pathology of the condition.

While experts throughout Europe agree on many aspects of the laboratory diagnosis of Fabry disease (for a recent review see Winchester and Young 2010), there are some specific issues which need further discussion before a general recommendation can be made. In order to promote this process, three one-and-a-half day ad hoc meetings* of European experts** were organized in 2009 with the aim of defining a consensus on laboratory diagnostics of Fabry disease that should allow for laboratories performing this diagnostic service to work to the same standards. Invitations to attend the meetings were based on, in addition to geographic considerations, outstanding scientific accomplishments in the field and/or active involvement in the laboratory diagnostics of Fabry disease.

*'Diagnostic use and value of Gb3 and lysoGb3 in Fabry disease' (April 22-23, 2009, Bad Nauheim, Germany); 'Diagnostic use and value of measuring alpha-galactosidase A activity in Fabry disease' (July 16-17, 2009, Berlin, Germany); 'Diagnostic use and value of mutations of the GLA gene in Fabry disease' (November 3-4, 2009, Barcelona, Spain). **J.M.F.G. Aerts (Amsterdam, The Netherlands), M. Beck (Mainz, Germany), O. Bodamer (Salzburg, Austria), A. Cooper (Manchester, United Kingdom), A. Gal (Hamburg, Germany), D. Germain (Paris, France), R. Giugliani (Zürich, Switzerland), D. Hughes (London, United Kingdom), L. Kuchar (Prague, Czech Republic), J. Ledvinova (Prague, Czech Republic), Z. Lukacs (Hamburg, Germany), C. Navarro (Vigo, Spain), E. Paschke (Graz, Austria), M. Piraud (Lyon, France), A. Rolfs (Rostock, Germany), C. Sa Miranda (Porto, Portugal), M. van Slegtenhorst (Rotterdam, The Netherlands), H. Treslova (Prague, Czech Republic), M.T. Vanier (Lyon, France), F.W. Verheijen (Rotterdam, The Netherlands), B. Winchester (London, United Kingdom). 
Primary objectives of the initiative were (i) establishment of 'gold standards' and practical algorithms for diagnosis of Fabry disease, (ii) promotion of a concept of certification/accreditation for diagnostic and treatment centres of excellence, and (iii) provision of a platform for future treatment guidelines. In particular, three specific issues were selected by the organizers: the diagnostic utility of measurement of $\mathrm{Gb} 3$ and lysoGb3, evaluation of $\alpha$ galactosidase A activity, and analysing mutations of the GLA gene in patients with Fabry disease. The most important goal of these workshops was to provide a platform for the experts to exchange ideas and experiences and share experimental data with each other, even if preliminary or yet unpublished, on the use, practical value, and significance of different laboratory approaches in the diagnostics of Fabry disease. All data were critically discussed by the panel and used to prepare this recommendation for the medical community. We hope that this document will serve as a starting point for further discussion that, in the end, should result in a consensus, provide essential guidance to physicians, and ensure a better differential and timely diagnosis of patients with Fabry disease.

\section{Diagnostic use and value of measuring $\alpha$-galactosidase A activity}

In normal human tissues, there are two lysosomal glycosidases with $\alpha$-galactosidase activity towards synthetic substrates. $\alpha$-Galactosidase A ( $\alpha$-GAL; EC 3.2.1.22) which is deficient in Fabry disease, acts on terminal $\alpha$ galactosyl residues in glycosphingolipids, whereas the so called $\alpha$-galactosidase B is an $\alpha-N$-acetylgalactosaminidase $(\alpha-N A G A L ; E C ~ 3.2 .1 .49)$ that acts on natural substrates with terminal $\alpha-N$-acetylgalactosaminyl residues and is defective in Schindler disease. $\alpha$-Galactosidase A does not catalyse the hydrolysis of the natural substrates of $\alpha$ NAGAL whereas $\alpha$-NAGAL may act on some natural substrates with terminal $\alpha$-galactosyl residues (Clark and Garman 2009). As both enzymes act on synthetic $\alpha$ galactoside substrates, $\alpha$ - $N$-acetylgalactosamine, a specific inhibitor of $\alpha$-NAGAL is added to assays of $\alpha$-galactosidase A activity for the diagnosis of Fabry disease. The genes (GLA and NAGA) encoding $\alpha$-GAL and $\alpha$-NAGAL are on chromosomes Xq22.1 and 22q13, respectively. Although the two genes show considerable homology, as they evolved from a common ancestral precursor (Clark and Garman 2009), $\alpha$-galactosidase $\mathrm{A}$ and $\alpha$-NAGAL have distinct physicochemical properties, and antibodies raised against one do not cross-react with the other.

$\alpha$-Galactosidase A is a typical lysosomal hydrolase with optimal activity towards natural and synthetic substrates at $\mathrm{pH} 3.8-4.6$. It is a glycoprotein and is transported to the lysosomes via the mannose-6-phosphate pathway. It is synthesized as a precursor of $50 \mathrm{kDa}$ and is processed to a mature lysosomal form of $46 \mathrm{kDa}$ by partial proteolysis and modification of its carbohydrates. The enzyme is a homodimer with an active site in each of the monomers. Hydrolysis of its natural substrates in vivo requires saposin $\mathrm{B}$ whereas that in vitro requires the addition of a detergent, usually sodium taurocholate. Patients with a genetic deficiency of saposin B also accumulate the substrates for $\alpha$-galactosidase $\mathrm{A}$, as in Fabry disease. The threedimensional structure of recombinant human $\alpha$ galactosidase $\mathrm{A}$ has been determined by X-ray crystallography at a resolution of $3.25 \AA$, and used to understand the effects of mutations on the structure and function of the enzyme (Garman and Garboczi 2004, Garman 2007).

After thorough clinical evaluation, determination of $\alpha$ galactosidase A activity is the first step in the laboratory diagnosis of a patient suspected of having Fabry disease, unless there is a known familial GLA mutation, for which DNA analysis is straightforward (see later). The activity can be determined in various materials, such as plasma, leukocytes, fibroblasts, or dried blood spots (DBS); the assay is rapid, reliable, and cost-effective. Currently the assay of $\alpha$-galactosidase A activity in leukocytes represents the diagnostic 'gold-standard'. A skin biopsy is rarely taken for initial diagnosis but studies on fibroblasts could be useful for molecular characterization of the enzyme deficiency. The most commonly used method of enzyme analysis is based upon cleavage of 4-methylumbelliferyl- $\alpha$ D-galactoside, a synthetic fluorigenic substrate. Recently a substrate suitable to assay enzyme activity by electrospray ionization/tandem mass spectrometry (ESI/TMS) has also become available ( $\mathrm{Li}$ et al. 2004, Zhang et al. 2010). The activity of $\alpha$-galactosidase A should always be determined in the presence of $\alpha-N$-acetylgalactosamine, which inhibits $\alpha$-galactosidase B. In addition, another lysosomal enzyme, preferably $\beta$-galactosidase, should always be assayed to evaluate sample quality. As enzyme activity may diminish rapidly if the sample is inappropriately stored and/or transported, the diagnostic laboratory should be contacted in advance for information regarding appropriate sample storage and shipping conditions. About $2 \%$ of the population/Fabry patients carry the non-synonymous change c.937 $\mathrm{G}>\mathrm{T}$ (p.Asp313Tyr). This variant has low $\alpha$-galactosidase A activity in plasma compared to the wild-type and about $60 \%$ of the mean $\alpha$-galactosidase A activity of the wild-type in cells. The p.Asp313Tyr variant is stable at lysosomal $\mathrm{pH}$ and is not disease-causing (pseudodeficiency).

Males with the classic Fabry disease phenotype can be reliably diagnosed by detecting complete deficiency or only negligible ( $<5 \%$ of mean normal) $\alpha$-galactosidase A activity. Single male patients with attenuated phenotypes may show considerable residual enzyme activities (e.g. 
Gaspar et al. 2010) that are still, however, well below the normal reference range. If initial enzyme activity analysis is performed on DBS of a male proband and shows pathologic values, a confirmatory assay, preferably on leukocytes, is recommended. For the index patient of any family with a biochemically proven $\alpha$-galactosidase $\mathrm{A}$ deficiency, mutation analysis of the GLA gene should be offered. This test can confirm the diagnosis and may provide additional information for disease prognosis and therapy. It is essential when genetic counselling of family members is desired (see later).

Identification of heterozygous females is not reliably made by the measurements of $\alpha$-galactosidase A activity because of the significant levels of activity that may be present in these samples due to random X-inactivation. Thus for females, a number of complementary diagnostic approaches is used currently, including measurement of urinary/plasma storage products, and histology/electron microscopy. However, only the identification of a diseaserelevant heterozygous GLA mutation allows definite diagnosis of carrier status.

Neonatal screening for Fabry disease in males is technically feasible by measurement of $\alpha$-galactosidase A activity in DBS using either the fluorigenic or mass spectrometric substrate and will detect cases both with complete deficiency and residual enzyme activity (Lin et al. 2009). High-throughput measurement of $\alpha$-galactosidase $A$ activity in leukocytes and DBS, for example by the technique of ESI/TMS, has been used to identify patients with Fabry disease in cohorts with advanced organ manifestations typical for Fabry disease (high-risk populations; for a recent review see Linthorst et al. 2010). It has been reported that $\beta$-glucuronidase activity is elevated in Fabry patients (Z. Lukacs, personal communication). Therefore the ratio of $\alpha$-galactosidase $A$ to $\beta$ glucuronidase activities may be helpful for increasing diagnostic discrimination, particularly for heterozygotes in these populations.

\section{Diagnostic use and value of Gb3 and lysoGb3}

Gb3 (Gb3Cer, globotriaosylceramide), also known as GL3 or $\mathrm{CTH}$, is a substrate of $\alpha$-galactosidase $\mathrm{A}$ and the main glycosphingolipid which accumulates when there is a deficiency of the enzyme. It may therefore be useful in diagnosis and assessment of disease burden. Deacylated Gb3, globotriaosylsphingosine (lysoGb3Cer or lysoGb3), is a minor metabolite recently identified in plasma of patients with Fabry disease. LysoGb3 might be more directly involved in the pathology and may also be useful in monitoring the disease (Aerts et al. 2008). Gb3 profiling (comparative analysis of various Gb3 isoforms) rather than measurement of the total urinary Gb3 concentrations, may represent a new diagnostic avenue in the future.

For biochemical analysis, Gb3 is easily accessible in various body fluids, including plasma and urine, and there are a number of validated assays including HPLC, TLC and LC/MS. In general, Gb3 should be measured in urine rather than in plasma, whereas lysoGb3 should be determined in plasma (Young et al. 2005, Togawa et al. 2010). Samples need to be sent fresh to the laboratory or should be frozen and subsequently transported frozen. It is recommended that both the biochemical analysis of lysoGb3 and Gb3 in plasma and urine, respectively, as well as the metabolite analysis are performed in specialized centers with appropriate experience. Gb3 inclusions can also be detected in cells of various tissues and organs, e.g. by biopsy of the conjunctiva, skin, kidney or heart, by electron microscopy and histochemistry including immunohistochemistry.

Plasma Gb3 levels do not correlate with disease manifestations: They are elevated in males with Fabry disease but are normal or only mildly elevated in females. Since Gb3 seems to be directly involved in the renal pathology of Fabry disease as a result of vascular compromise, podocyte toxicity, and focal tubular damage and sclerosis, quantification of urinary Gb3, that is derived primarily from tubular epithelial cells, is a possible diagnostic procedure for patients presenting with the classic Fabry disease phenotype (Sessa et al. 2003). A relationship between urinary Gb3 levels and Fabry disease severity as well as to therapeutic response has been demonstrated in a few studies (Whitfield et al. 2005, Banikazemi et al. 2007, Eng et al. 2001, Schiffmann et al. 2001). More recent studies suggest that there is no linear relationship between urinary Gb3 concentrations and end-organ effects of Fabry disease in male or in female patients. Furthermore it is currently unclear whether or not increased urinary Gb3 concentration can be taken as a specific marker for diseaserelated lysosomal storage. Whilst there is no doubt that Gb3 accumulates in patients with Fabry disease, from placental development onwards (Vedder et al. 2006), its role in the assessment or management of patients with this condition is contentious.

While single assessments of Gb3 add only very little to the diagnosis of patients with biochemically and/or clinically ascertained Fabry disease, serial measurements of total urinary Gb3 (plasma lysoGb3) in the same individual may be used to monitor the progression of the pathological process. In male patients, a response to ERT is indicated by a decline of urinary Gb3, whereas an increase after prolonged enzyme administration may point to the existence of interfering antibodies against infused $\alpha$ galactosidase A (Schiffmann et al. 2006, Vedder et al. 2007, Hughes et al. 2008). Analysis of total urinary Gb3 from a 12-24 h urine sample may be a diagnostic adjunct in 
females suspected of having Fabry disease when no male index patient is available for mutation analysis.

In symptomatic males with Fabry disease, plasma lysoGb3 is elevated probably from an early age, and is strikingly high in adult male patients, although no correlation has been found between plasma lysoGb3 and disease manifestations (Aerts et al. 2008). In females, plasma lysoGb3 levels increase steadily and show a weak correlation with disease manifestation. Provided that comprehensive analyses of plasma lysoGb3 data from large cohorts of patients with Fabry disease confirm the preliminary observations, lysoGb3 may prove to be a promising novel diagnostic tool (Rombach et al. 2010). Where gene alterations are found with unclear functional or pathological implications in females, the assessment of Gb3 metabolites/isoforms (urinary Gb3 and plasma lysoGb3) is recommended.

\section{Diagnostic use and value of mutations of the $G L A$ gene}

The GLA gene, encoding $\alpha$-galactosidase A, contains seven exons ranging from 92 to 291 base pairs (bp) in length. The coding region consists of $1,290 \mathrm{bp}$ and encodes a polypeptide of 429 amino acids, with the first 31 residues representing a signal sequence. As of 30th of June 2009, a total of $599 G L A$ sequence changes, including 435 probable pathogenic point mutations (missense, nonsense, and splice site) and 150 disease-causing 'short length' rearrangements (mainly deletions and duplications affecting less than 65 nucleotides) as well as 14 DNA polymorphisms have been reported (for a recent review see Gal 2010). Large rearrangements involving one or more exons or the whole GLA gene seem to be infrequent (ca. 5\%) in patients with Fabry disease. Most of the GLA mutations found in patients with Fabry disease are novel (unique, 'private'). The frequency of de novo mutations has been estimated to be $3-10 \%$. There are a few reports of patients carrying two different, highly probable disease-causing GLA mutations on the same allele. In contrast, patients may carry one or more of the common GLA polymorphisms, including several non-synonymous changes, or rare non-pathogenic variants, in addition to their disease-causing mutation. To date, no experimentally validated data have been presented that any of these latter sequence variants, alone or in combination, are disease-causing.

As a practical approach one can define two classes of mutations to assess their pathogenic significance. Class 1 mutations are predicted to have high probability of causing disease because they create a premature stop codon (nonsense or frame-shift mutations) with the consequent loss of the protein, affect the evolutionarily conserved splice-site dinucleotides at the beginning or the end of one of the $6 G L A$ introns and interfere with the proper splicing of the GLA mRNA, or severely disrupt the coding sequence by a large rearrangement. Sequence changes resulting in a catalytically inactive (non-functional) enzyme, for example missense mutations affecting one of the 15 residues in the enzyme active site, or one of the 8 cysteines essential for proper three-dimensional protein folding, are also class 1

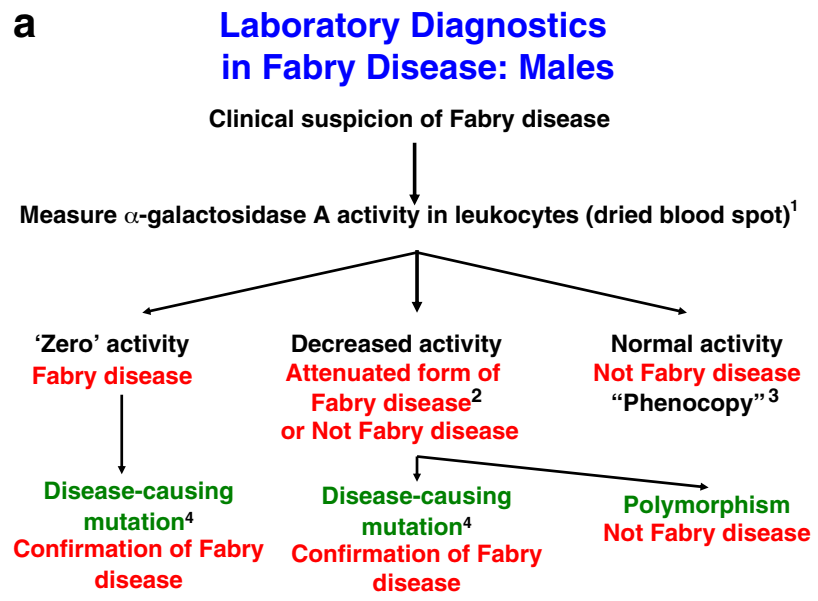
b Laboratory Diagnostics in Fabry Disease: Females

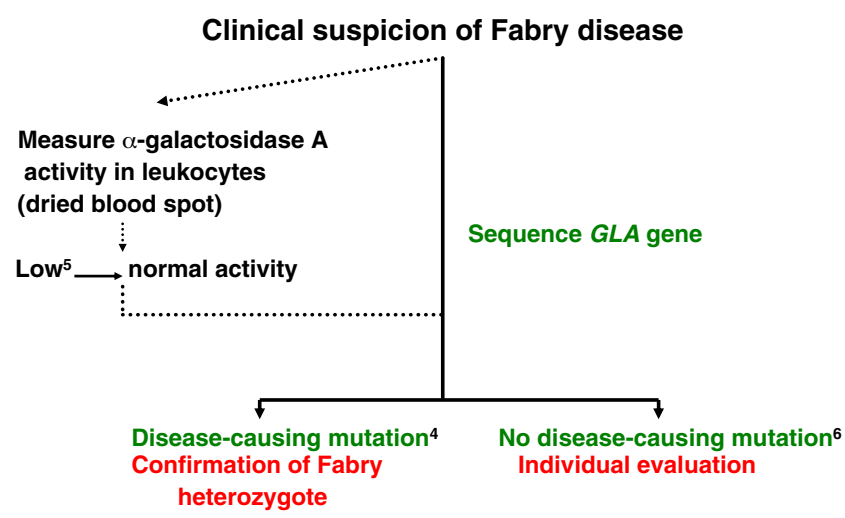

Fig. 1 Algorithm for the laboratory diagnosis of (a) male and (b) female patients with Fabry disease. The flow charts represent summaries of the discussion and conclusions in the text, with extra clarification in points 1-6. 1) Demonstration of a deficiency of $\alpha$ galactosidase $\mathrm{A}$ in leukocytes and plasma from the same blood sample is supporting evidence for a diagnosis of Fabry disease. 2) Variants of Fabry disease with residual $\alpha$-galactosidase A activity. 3) A patient with some symptoms of Fabry disease not due to deficiency of $\alpha$ galactosidase A. 4) Class 1 mutation and mutations previously found in affected male or female Fabry patients. 5) Low $\alpha$-galactosidase A activity can be suggestive of carrier status but not definitive. 6) To decide whether the proband has Fabry disease, all or some of the following investigations should be carried out: (a) re-evaluation of clinical presentation, (b) analysis of family pedigree, (c) measurement of urinary $\mathrm{Gb3}$, and (d) in absence of a Class 1 mutation, any sequence change detected in the GLA gene must be expressed in vitro to investigate its effect on activity and/or structure of enzyme 
mutations. Finally, recurrent disease-causing mutations and the growing number of GLA mutations that have been shown to result in non-functional enzyme in in vitro expression studies in cells in cultures transduced with the relevant mutant cDNA also belong to this group. It has been suggested that the pathology of some 'simple' disease-causing mutations might occasionally be complex. Indeed, some DNA changes thought to be missense mutations (e.g. p.Ser65Thr, p.Gly183Ser, p.Lys213Asn, or p.Met267Ile) seem to interfere with normal splicing of the GLA mRNA (Lai et al. 2003). Similarly, two deep intronic point mutations (c. $639+861 \mathrm{C}>\mathrm{T}$ and c. $639+919$ $\mathrm{G}>\mathrm{A}$ ) apparently result in $\alpha$-galactosidase A deficiency by causing complex changes in the pattern of splicing (Ishii et al. 2002, Filoni et al. 2008). About $50 \%$ of males with enzymatically proven Fabry disease carry a Class 1 mutation.

Mutations that have been found in males with normal $\alpha$ galactosidase A activities or with somewhat decreased enzyme activities, which are still, however, well above the pathologic range of values, are called Class 2 mutations and are almost certainly non-pathogenic. These include polymorphisms or sequence changes, e.g. c.937G $>$ T (p.D313Y) described as pseudodeficiency alleles.

Routine mutation analysis of the GLA gene consists of sequencing of the coding region and exon-intron boundaries. The interpretation of gene alterations should be performed by an expert and genetic counselling should be offered. In more than $97 \%$ of males with pathologic $\alpha$ galactosidase $\mathrm{A}$ activities, a sequence variant (Class 1 mutations or yet unclassified sequence changes) can be detected by routine mutation analysis. The identification of Class 1 mutations is considered a very useful and independent confirmation of the biochemical (and clinical) diagnosis. A small number of mutations, in particular exon-spanning duplications or inversions and deep intronic mutations, may escape detection by using the above method and can only be identified by more sophisticated procedures, such as the analysis of the mRNA or MLPA (multiplex ligation-dependent probe amplification). However, even if no highly probable disease-causing mutation is found in a male patient with an $\alpha$-galactosidase A deficiency, the diagnosis of Fabry disease remains valid. For mutations not categorized as Class 1 or Class 2, a thorough clinical, biochemical and genetic analysis of the patient and his family may allow designation of the mutation as disease-causing or not. Analysis of evolutionary conservation of the relevant sequence and its absence in a large cohort of unaffected individuals may provide additional arguments for a pathogenic relevance of a change. An easily accessible and quality-controlled gene-specific mutation database would be an invaluable tool both for physicians treating patients, genetic counselors, and scientists involved in DNA diagnostics or research.

Both Class 1 and Class 2 mutations also occur in females. Since enzyme activity measurements do not reliably detect heterozygotes, and many of the clinical features of Fabry disease are frequently observed in the general population, DNA diagnostics is much less efficient in identifying heterozygotes among women with clinical suspicion for Fabry disease than in confirming diagnosis in males with enzymatically proven Fabry disease. Carriers in families with Fabry disease can be identified by pedigree analysis and/or by showing that they have inherited the family-specific mutation. If bidirectional sequencing of the coding region and exon-intron boundaries do not reveal heterozygosity for a Class 1 mutation in a female DNA sample, MLPA may help to pick up the few cases of large DNA rearrangements in females, whereas the analysis of mRNA may be complicated by nonsense-mediated decay. In addition, a thorough clinical re-evaluation of the patient and additional biochemical tests are recommended. A careful analysis of the pedigree by a genetic counselor should also be offered, keeping in mind that nine out of ten patients with Fabry disease should have a positive family history for the trait. In females with typical signs and symptoms of Fabry disease but without positive family history, only the identification of a disease-relevant heterozygous GLA mutation allows definite diagnosis of carrier status.

Conclusions (Fig. 1): (i) Thorough analysis of the patient's family and medical history together with a clinical examination are essential prior to laboratory testing. (ii) Diagnostic tests should be done in laboratories with appropriate quality control schemes, experience, and sample load. (iii) Analysis of $\alpha$-galactosidase A activity is the standard diagnostic test of Fabry disease in males. Molecular analysis of the GLA gene is necessary to diagnose heterozygotes.

Acknowledgments The European Consensus on Diagnostics in Fabry Disease was sponsored by an unrestricted education grant generously awarded by The Center for Extramural Clinical Research and Education of Shire Human Genetic Therapies, Inc.

Open Access This article is distributed under the terms of the Creative Commons Attribution Noncommercial License which permits any noncommercial use, distribution, and reproduction in any medium, provided the original author(s) and source are credited.

\section{References}

Aerts JM, Groener JE, Kuiper S et al. (2008) Elevated globotriaosylsphingosine is a hallmark of Fabry disease. Proc Natl Acad Sci USA 105:2812-2817

Banikazemi M, Bultas J, Waldek S et al. (2007) Agalsidase-beta therapy for advanced Fabry disease: A randomized trial. Ann Intern Med 146:77-86 
Clark NE, Garman SC (2009) The 1.9 a structure of human alpha-Nacetylgalactosaminidase: the molecular basis of Schindler and Kanzaki diseases. J Mol Biol 393:435-447

Eng CM, Guffon N, Wilcox WR et al. (2001) Safety and efficacy of recombinant human alpha-galactosidase A-replacement therapy in Fabry's disease. N Engl J Med 345:9-16

Filoni C, Caciotti A, Carraresi L et al. (2008) Unbalanced GLA mRNAs ratio quantified by real-time PCR in Fabry patients' fibroblasts results in Fabry disease. Eur J Hum Genet 16:13111317

Gal A (2010) Molecular genetics of Fabry disease and genotypephenotype correlation. In Elstein et al. eds. Fabry disease. Springer Science + Business Media B.V. pp. 3-19

Garman SC (2007) Structure-function relationships in alphagalactosidase A. Acta Paediatr 96(Suppl 455):6-16

Garman SC, Garboczi DN (2004) The molecular defect leading to Fabry disease: structure of human alpha-galactosidase. J Mol Biol 337:319-335

Gaspar P, Herrera J, Rodrigues D et al. (2010) Frequency of Fabry disease in male and female haemodialysis patients in Spain. BMC Med Genet 11:19-26

Hughes DA, Elliott PM, Shah J et al. (2008) Effects of enzyme replacement therapy on the cardiomyopathy of Anderson-Fabry disease: a randomized, double-blind, placebo-controlled clinical trial of agalsidase-alfa. Heart 94:153-158

Ishii S, Nakao S, Minamikawa-Tachino R, Desnick RJ, Fan JQ (2002) Alternative splicing in the $\alpha$-galactosidase A gene: increased exon inclusion results in the Fabry cardiac phenotype. Am J Hum Genet 70:994-1002

Lai LW, Whitehair O, Wu M-J, O'Meara M, Lien Y-H H (2003) Analysis of splice-site mutations of the $\alpha$-galactosidase A gene in Fabry disease. Clin Genet 63:476-482

Li Y, Scott CR, Chamoles NA et al. (2004) Direct multiplex assay of lysosomal enzymes in dried blood spots for newborn screening. Clin Chem 50:1785-1796

Lin HY, Ching KW, HSu JH et al. (2009) High incidence of the cardiac variant of Fabry disease revealed by newborn screening in the Taiwan Chinese population. Circ Cardiovasc Genet 2:450-456
Linthorst GE, Bouwman MG, Wijburg FA, Aerts JM, Poorthuis BJ, Hollak CE (2010) Screening for Fabry disease in high-risk populations: a systematic review. J Med Genet 47:217-222

Rombach SM, Dekker N, Bouwman MG et al. (2010) Plasma globotriaosylsphingosine: diagnostic value and relation to clinical manifestations of Fabry disease. Biochim Biophys Acta May 13. [Epub ahead of print] PubMed PMID: 20471476

Schiffmann R, Kopp JB, Austin HA et al. (2001) Enzyme replacement therapy in Fabry disease: a randomized controlled trial. JAMA 285:2743-2749

Schiffmann R, Ries M, Timmons M, Flaherty JT, Brady RO (2006) Long-term therapy with agalsidase alfa for Fabry disease: safety and effects on renal function in a home infusion setting. Nephrol Dial Transplant 21:345-354

Sessa A, Meroni M, Battini G et al. (2003) Studio multicentrico Italiano sulla malattia di Anderson-Fabry. Evolution of renal pathology in Fabry disease. Acta Paediatr 92 (Suppl 443): 6-8; discussion 5. PubMed PMID: 14989458

Togawa T, Kodama T, Suzuki T et al. (2010) Plasma globotriaosylsphingosine as a biomarker of Fabry disease. Mol Genet Metab Apr 1. [Epub ahead of print] PubMed PMID: 20409739

Vedder AC, Strijland A, vd Bergh Weerman MA, Florquin S, Aerts JM, Hollak CE (2006) Manifestations of Fabry disease in placental tissue. J Inherit Metab Dis 29:106-111

Vedder AC, Linthorst GE, van Breemen MJ et al. (2007) The Dutch Fabry cohort: diversity of clinical manifestations and Gb3 levels. J Inherit Metab Dis 30:68-78

Whitfield PD, Calvin J, Hogg S et al. (2005) Monitoring enzyme replacement therapy in Fabry disease - role of urine globotriaosylceramide. J Inherit Metab Dis 28:21-33

Winchester B, Young E (2010) Laboratory diagnosis of Fabry disease. In Elstein et al. eds. Fabry disease. Springer Science + Business Media B.V. pp. 111-132

Young E, Mills K, Morris P et al. (2005) Is globotriaosylceramide a useful biomarker in Fabry disease? Acta Paediatr 94:51-54

Zhang XK, Elbin CS, Turecek F et al. (2010) Multiplex lysosomal enzyme activity assay on dried blood spots using tandem mass spectrometry. Methods in molecular biology 603:339-350 